\title{
A Novel Method of an Online Opinion Mininge Motions by Comparing Various Mobile Gadgets
}

\author{
K.Gurumoorthy, P.Suresh
}

\begin{abstract}
The drastic development in the web world gives a greater impact through various sources like online journals, e-commerce sites, peer-to-peer networks and social media, consumers have a broad platform and unlimited power to share their experiences in the form of reviews. With this, majority of reviews are accessible for a solitary item, that should be investigated, handled and mine. Opinion Mining or Sentiment Analysis is a Natural Language Processing and Information recovery assignment that characterizes the client's perspectives or assessments through positive, negative or unbiased conclusions. Aspect based Opinion Mining manages parts of the highlights. In this paper an aspect based sentiment mining framework is suggested that order reviews as positive and negative. With the help of the SVM the accuracy can be find out to get the clear idea of the reviews. Moreover four brands will discuss in our research. The various emotions, positive and the Negatives will be analyzed clearly in this paper. The customer review, rating by the customers, rate comparing and the opinion mining will be explained in the last section. The framework additionally manages two viewpoints in a survey. Experimental results using reviews of mobile phones show an accuracy of $86 \%$ as compared to other methods.
\end{abstract}

\section{Keyword : SVM,Sentiment,Phishing,Clustering}

\section{INTRODUCTION}

Sentimental Analysis (SA) is a system which forms subjectivity and opinions of the content to draw imperative viewpoint around an element and gives an abridged decision about the information, which can be additionally utilized for basic leadership. Various types conclusions are delegated: : and their frequency, part of speech, sentiment words and phrases, rules of syntactic dependency, sentiment shifters and opinions.A lot of data shared on mobile devices has opened space for another influx of portable applications. There is a vast potential for opinion investigation strategies for portable conditions and the vast majority of the exertion has been focused on the advancement of methodologies ready to

quantify the prosperity of a mobile phone client $[1,2,3]$.

There are numerous different applications, for instance, to enable clients to sort out the data they read and even break down suppositions separated from the substance they get [4]. Be that as it may, little is thought about the deploy ability of these techniques in the versatile condition. Actualize opinion examination innovation on mobile devices is key for some

Revised Manuscript Received on September 22, 2019.

K.Gurumoorthy, Research Scholar, Periyar University, Salem, Tamilnadu, India

Dr.P.Suresh, Research Supervisor,HOD, Dept. of Computer Science,Salem Sowdeswari College, Salem, Tamilnadu. applications. Initially, sentence-level conclusion investigation strategies speak to the reason for some different techniques to quantify client mind-set and emotions. Second, it enables applications to gauge slant of client's texts.

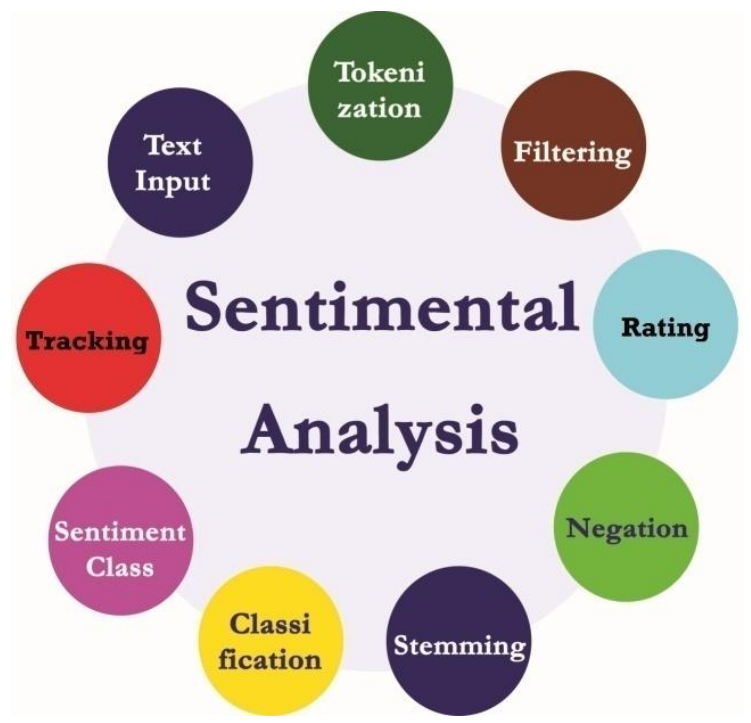

Fig 1: Architecture of Sentiment Analysis

Content mining is a specific space that applies information mining procedures over content. Assumption examination plans to distinguish and remove opinions, states of mind and moods of individuals and communities .The authors provide [5] a specialized review and early work on notion investigation. At the web based networking media information, the outcome is regularly a ground-breaking elucidating or prescient device; in [6], content mining was fruitful connected to separate Twitter posts for assumption order for item wellbeing in medicinal field.

Assessment investigation of web-based social networking information has additionally be connected for rating the items as talked about by the creators of [7]. Each creator proposes their own strategies to assess suppositions. Sadly, the majority of the opinion examination instruments or calculations are as yet being looked into. At present, there is no calculation that can give a hundred percent exact outcomes for opinion investigation. There are as yet a few discussions going ahead between different specialists endeavoring to demonstrate that their answer is more immaculate than the others. 


\section{LITERATURE}

Li, Xiaodong et al., [8] proposed thoughtful trade making sense of how to trade the data picked up from news-rich stocks that are inside a comparative fragment to the news-poor stocks. News stories of the two sorts of stocks are mapped into a comparable segment space that is worked by end estimations. New markers are then arranged in the nostalgic space rather than the standard ones. Dataset used is Hong Kong stock.

Al-Sarhan, Huda, et al., [9] Aims to developing the space of Arabic brimming with supposition new examination through giving (an) a benchmark explained Arabic dataset of news for loaded with conclusion news assessment (b) a perspective based feeling examination (ABSA) approach for surveying the nostalgic impact of Arabic news posts on the peruser, and (c) a standard technique with a run of the mill appraisal framework to differentiate future research results and the measure ones. Dataset: During the strikes of Gaza which prop up for four months, the immense events in the midst of this period are parceled into eight subintervals based of the centrality of the event. The netvizz toll has been used to crawl news posts from facebook pages called breaking news from GAZA.

Bouazizi et al., [10] proposed an increasingly significant model built methodology of collection regarding information of twitter; the request is done into 7 particular classes giving twofold plan and ternary portrayal and associated multi-class supposition examination.

Perform appraisal is done on dataset assembled from Yelp. The result of this examination shows well portray customer tendencies sentences for the improved execution proposition.

Mother, Hongxia et al., [13] presents a cross-vernacular evaluation request methodology reliant on SVM. Used real strategies evacuate incorporate words in English and use machine elucidation word weighting technique joined TF-IDF with thoughtful intensity of estimation words then a vector space show is created. Dataset: English clarification data of three areasDVD, book and music with $2000+$ vet and ve models, little proportion of Chinese remark enlightening list of $20+v e$ and - ve points of reference Desai et al., [14] proposed a framework which join shallow parsing and jargon estimation, that pick space and perspective base examination of assessment and a short time later furthest point is consigned to a lexicon. constituents words are aggregated by separating sentence using the shallow parsing system.

Liu, Jie et al., [15] through and through examination of overviews on JD.com watched the period of postings, measurement of customers on the other had customers need to post short surveys containing the delineation of significant worth and cost of the thing. Feelings and ends is one of the key factors that can persuade the audits quality. Selvan et al., [16] focused on supposition burrowing for the fastfeedback, the computational structure considering the complexity of tolerating reactions from customer which is a trying task while encountering surrey programming. The edge work in this paper has used progressing data stream of twitter.

Hagenau et al., [17] Enhances substance mining showed systems by techniques for extra preliminary features of substance address and by make use of analysis of market as a bit of our strategy of feature decision. Enlightening file are picked disseminated messages through trading hours and focused on zenith 110 essentially liquid stocks.Xianghua, $\mathrm{Fu}$ et al., [18]Global subject at first associated LDA(latest Dirichlet Allocation) model to discover than neighborhood proposed as un oversaw advance to discover normally the Chinese social relations analyzed perspectives and moreover inclinations explained in different viewpoints,

Moraes, Rodrigo et al.,[19]An preliminary appraisal among ANN and SVM as for idea assessment at record level, discussed need coming about settings and models in which two techniques achieve upgraded request measurements of all rally. Dataset: Four datasets, 1.Movie review dataset, 2.collection of Amazon.com studies mine specifically thing zones BOOKs, Cameras and GPS. Gotten standard advancement content with understood specific procedures for features assurance and weighing is a standard model of pack of words.ANN thing better/comparable result in assessment mind quantifiable broad complexity even some potential obstruction of the two models computational cost of running time SVM and planning time of ANN.

\section{BACKGROUND}

With the fast improvement of web, an ever increasing number of sorts of online data are accessible. In these data assets, there are bottomless of emotional remarks and perspectives, for instance, remarks for electronic items, vehicles, films, or reviews for a few occasions or strategies. It is important to choose whether the remarks are certain or negative, if the reviews are steady or oppositive, and if an item is recommendable or not? These necessity evoked the exploration theme of slant investigation. In a few literary works, opinion examination is additionally referd as supposition mining. Its essential goal is to anticipate the nostalgic extremity of given setting by recognizing wistful constituents like wistful words, abstract sentences, and so forth.

Supposition investigation analyzes a wide assortment of writings, from sentences to archives, for example, gathering remarks, website pages, blog articles, item reviews, and motion picture surveys, and so forth. The undertaking of assumption arrangement is grouping the extremity of the communicated opinion [20], which can be certain or negative, steady or not, and so on. Different machine learning techniques have been brought into this undertaking. For instance, Turney [21] used web crawler to process semantic propensity. Hu et al [22] endeavored to arrange word into supposition and non-opinion terms. [18] investigated word relations for opinion characterization. Ache et al [23] connected three distinct strategies, i.e., Support outcome. In addition, for highlight weighting, they discovered that in opinion arrangement, Binary (nearness/ nonappearance of words) is superior to Term 
Frequency (TF). They gathered 1400 film remarks as examination informational collection, on which the most elevated exactness achieved $82.9 \%$.

We refer as Web Analysis as the arrangement of systems, strategies and tools used to extricate and comprehend the data on the Web. Regardless of whether most Web clients believe that the chief wellspring of data is the Web content, such information it is nevertheless only a little level of the information contained on the Web. A few takes a shot at this territory incorporate fields like deciding and deciphering the page connections [36], [37], [38], location of Web people group [38], examination of social gatherings [33], [24], and the improvement of Web hazard discovery procedures [34], [35]. These themes have increased noteworthy significance amid the most recent decade, spoken to by the extraordinary number of uses created to take care of these issues. A necessity for these applications is to have a model to speak to the connections on the Web. For instance, usually to speak to the Web as a coordinated chart: Vertex are hubs containing some relevant data, while the edges between vertex are viewed as coordinated connections. This model is utilized in the Page Rank calculation [37] where vertex are pages and edges speak to joins. While the past graphical proposition is useful for human comprehension, when utilized in the investigation of a huge number of hubs, it turns out to be fairly illogical. So said data its for the most part communicated as the contiguousness and occurrence network portrayals recently talked in .

In the wake of having said this, we proceed by quickly depicting two cases and urge you to audit the referenced works.

Phishing Detection: With the extension of monetary exercises in the Web, another issue has raised the multiplication of imitated locales that look for data for unlawful allocation. In view of this, creating apparatuses to distinguish unsafe locales has turned into a need. Models of these devices are the phishing location calculations for suspicious pages situated in the Web chart, which permit to describe genuine pages. This page portrayal shifts from an absolutely manual boycotting to the exacerbated weighting of chosen highlights. A portion of these qualities are the visual subject, page content, areas name, Google relegated Page Rank, period of space, suspicious characters in the URL, and appearance of known pictures. When an arrangement of qualities is characterized, a vector for each page is made, where each incentive in the vector relates to one of the attributes. At that point, it is conceivable to choose a criteria to locate the speculate pages. This criterion is more often than not the use of a limit to the speck result of the measurements esteems. Subsequent to assessing each page with the given criteria, an arrangement of suspicious pages is acquired. Here, depending the usage, at least one moves could be made:

Accordingly, regardless of whether the idea is basic, making a decent portrayal of the pages with the end goal that it doesn't depends in human supervision is definitely not a straightforward issue. A large portion of the real methodologies require human mediation to choose the genuine phishing pages from conceivable false positives.

Community Detection: With the expansion of the Web administrations numerous gatherings have discovered a simple access, and minimal effort media to advance their belief systems. A portion of these gathering philosophies can speak to a threat, and in this way there is an enthusiasm for distinguishing them and following their activities. This isn't the main situation where distinguishing Web people group is required, promoting, social investigation, and web search tools advancement likewise require an approach to take care of this issue. In its least complex frame, a network is a gathering of people firmly interrelated. Sentiment Pattern based Analysis is used for an analyzers [39].

\section{PROPOSED TECHNIQUE}

In our we used four brand namely Samsung galaxy A6,VIVO V9, Motto G6, Xiomi Redmi Y2 for our comparison. In the figure 3 we mention the common review of the various brands. But its very difficult to apply our proposed technique in a vast setup. It leads to various confusion and the analysis will not be narrow ended. So focus four brand comparisons as a sample to project the clear idea of the sentimental analysis.

The real data can be collected from the Flip kart and the Amazon web pages to know the current review and the feedback of the customers. Even though we have plenty of trendy mobiles in the market we choose a middle class range mobiles for our comparison. Because then only we can get a good number of reviews and handful of percentage feedback. If we choose higher brand of Apple I phone then we can get only a certain group of people for the corresponding reviews. In our proposed work we choose four major mobile phone for the data clustering.

The variation factor can be viewed as a proportion of the comparability in the feeling articulations at the pages. For instance, this will amass together pages that have low articulation of positive assessment and a considerable measure of anger. Conversely, the partition factor estimates the closeness in the aggregate assessment and feeling content communicated in the page i.e. this will assemble together pages by the amount of feeling content.

We have a formula for the partition technique to segregate the reviews in a much better way.

$$
\begin{aligned}
& S\left(f_{i}, P_{i}\right)=|\vec{C}| \\
& |\vec{C}|=\sqrt{C_{1}^{2}}+C_{2}^{2}+\ldots . .+C_{n}^{2}
\end{aligned}
$$

The partition technique stores the following data in each component.

$$
\begin{aligned}
& C_{0}=P_{1}-P_{2} \\
& C_{1}=n_{1}-n_{2} \\
& C_{2}=\theta_{1}-\theta_{2}
\end{aligned}
$$

Where p1 corresponds to the positive value in the page vector corresponds to the negative review of the customer; is the standard deviation of the component. Similar values have been calculated for the cluster representative in. 


\section{A Novel Method of an Online Opinion Mininge Motions by Comparing Various Mobile Gadgets}

\section{V.MECHANISM OF THE WORK}

The general mechanism of our proposed work focuses the online mobile reviews through the sentimental analysis. The data can be collected through various sources like online,web, web portal, retail review pages etc. The data's can be crawled in various segments according to the need of the hour. The preliminary data can be processed into a different clusters. In the cluster it can be mapped and inter connected with each elements. The statistical analysis of the product can be prepared and compose with the help of the reviews. The Statistical analysis clearly defines the mathematical analysis of the corresponding product.
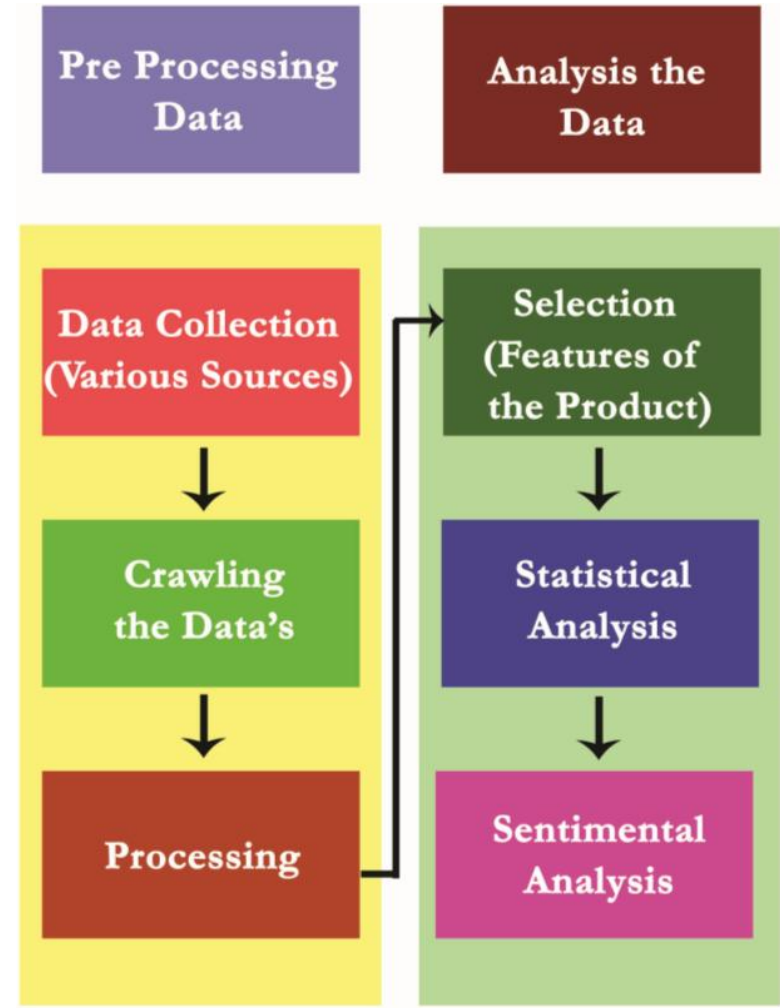

Fig 2: Flowchart of the proposed technique

In our research the mobile products can be reviewed and the analysis can be projected in a well manner. Finally the statistical analysis can be segregated and analyzed as a opinion way. The sentimental analysis projects the reviews in a various aspects of the viewers point. For eg., Joyful nature, sadness, anger, satisfactory, Positive, Negative reviews, Not satisfactory etc. Moreover the proposed technique focuses the module in various specific tasks. The data gathering, data processing, data clustering, data interpretation, data visulaisation.

\section{RESULTS}

The real data's can be taken from the flip kart, amazon and some other online review webpages for our proposed work. The major focus of the work is to cluster the reviews ( analyse) according to the opinion mining i.e) Sentimental analysis.

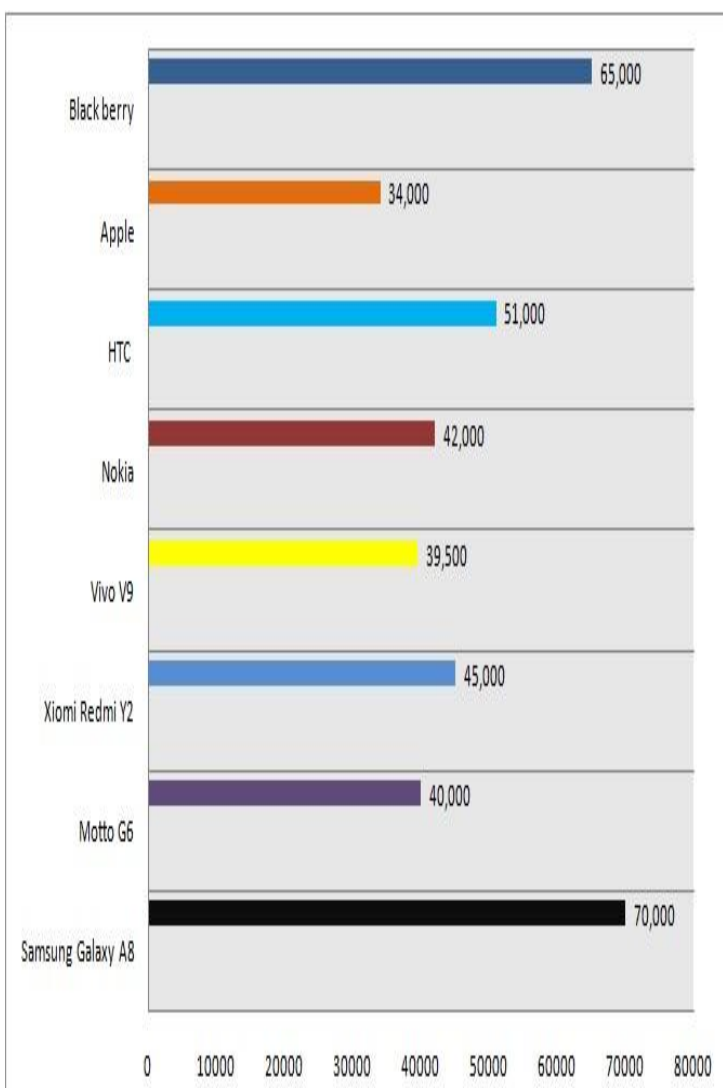

Fig 3: Reviews as per the various brand

In figure 3 the various reviews of the customers can be projected for various brands. The general comparision for the various brands can be defined in the above chart. Few of the brands can have the maximum number of reviews and the feedbacks because of the usage of the mobile phones. The blackberry and the Samsung have the maximum number of reviews by the customers. The blackberry can be used by certain group of people and it quite costly. Even though Samsung brand is costly we have plenty of varieties with lower cost. So people use these kinds of cost friendly mobiles and the reviews also maximum. In our proposed work we uses SVM machine to cluster and analyze the reviews

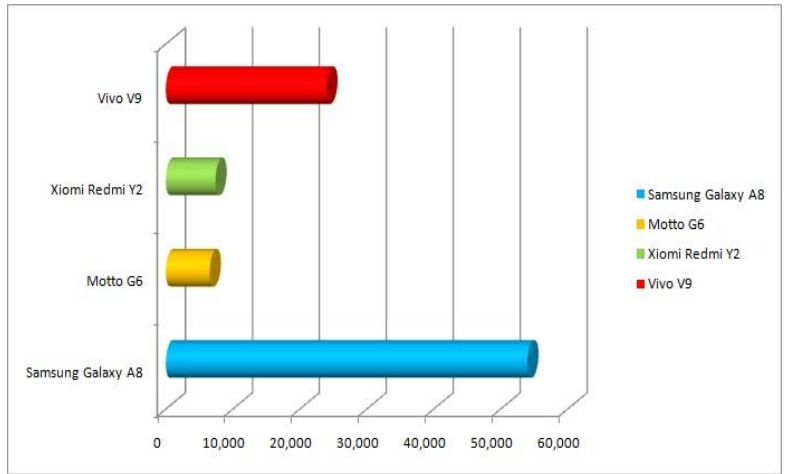

Fig 4: Brand reviews used in our proposed technique

The Specifically four brands can be compared and analyzed. Samsung galaxy A6 has the maximum reviews by the customers. The review contains the general feedback of the specific product. The general reviews contain the entire positive and the negative 
feedbacks. The same reviews can be taken up for the remaining three brands of mobile phones. The segregated reviews i.e) Sentimental analysis can be mentioned in the fig 6

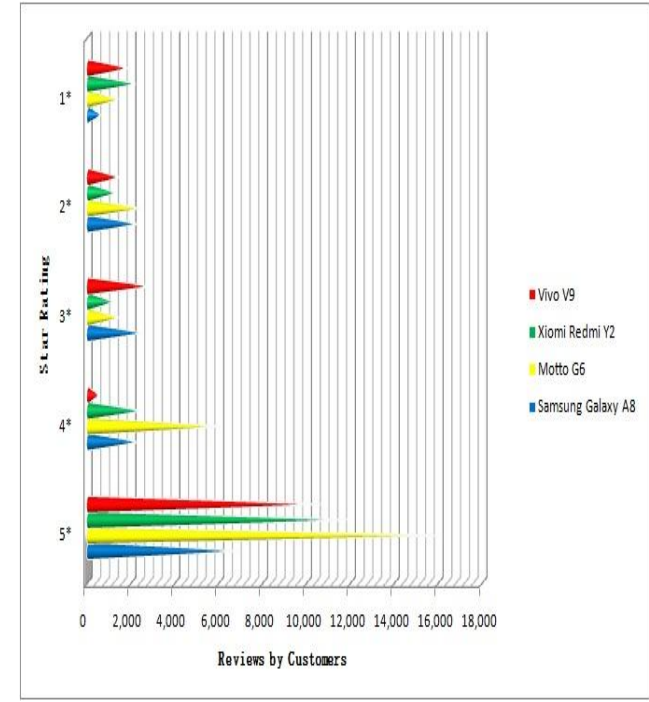

Fig 6: Star rating reviews

In figure 6 defines about the star rating of the each mobile brand. The rating contains the maximum and the minimum satisfactory process. The five star rating gives the positive feedback and the single star rating emphasise the minimum satisfactory condition.

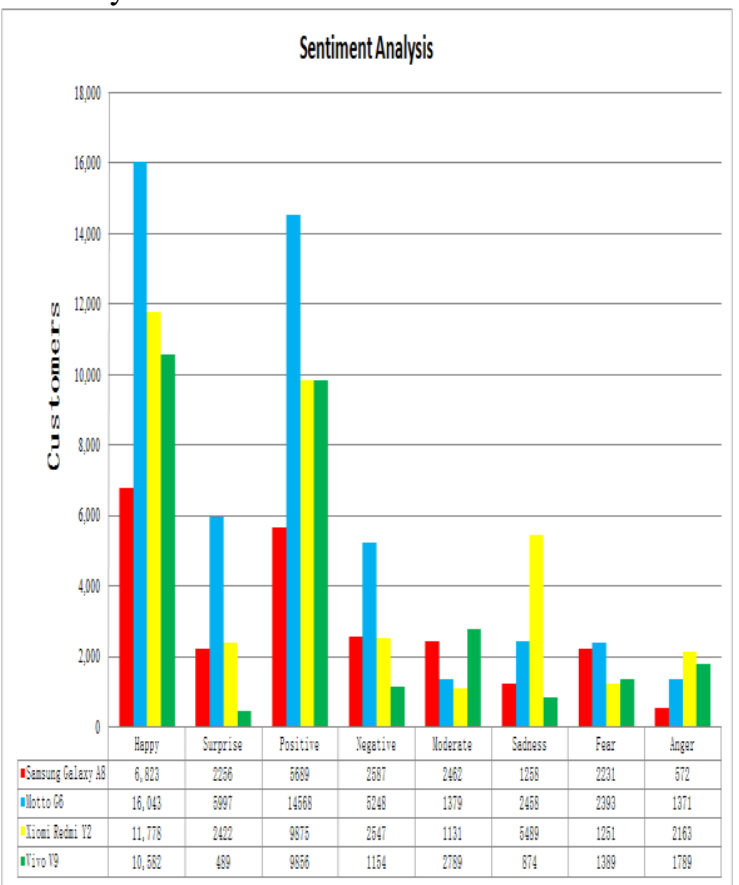

Fig 7: Sentimental analysis for the proposed mobile brands

In figure 7 we focus the sentimental analysis with various aspects of the reviews. The chart clearly explains about customer's mentality. The sentimental analysis can be carried out by the help of our proposed formula to cluster the reviews according to the opinion of the users. The real datas can be collected from the online review web pages to carry out the analysis. The star rating is one of the vital role to analyze the reviews in a crystal clear manner.

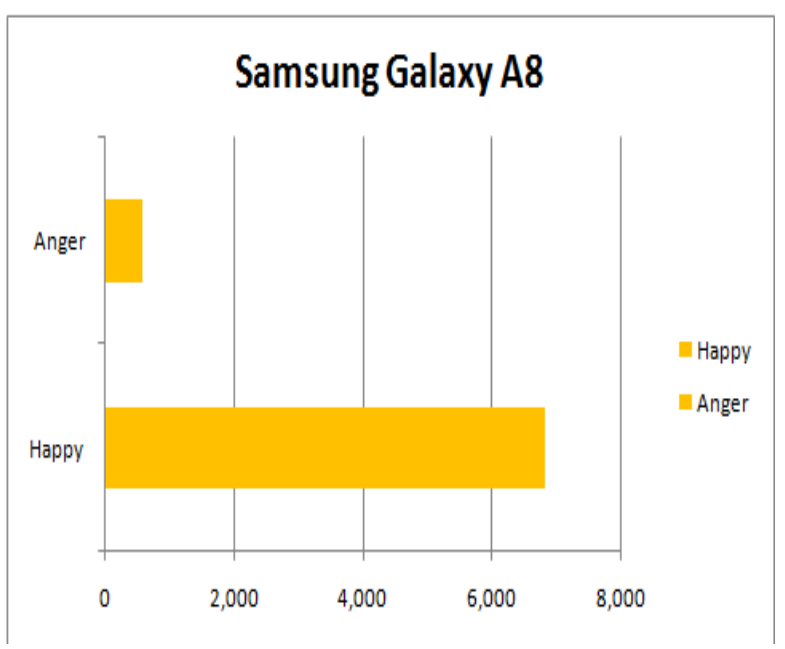

Fig 8: Positive and Negative reviews for Samsung

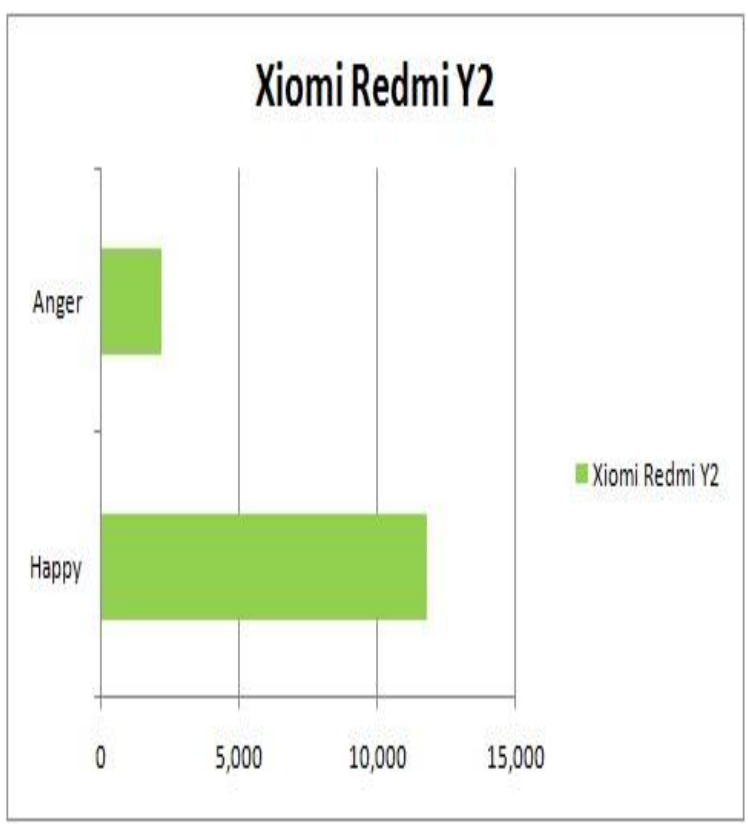

Fig 9: Positive and Negative reviews for Motto

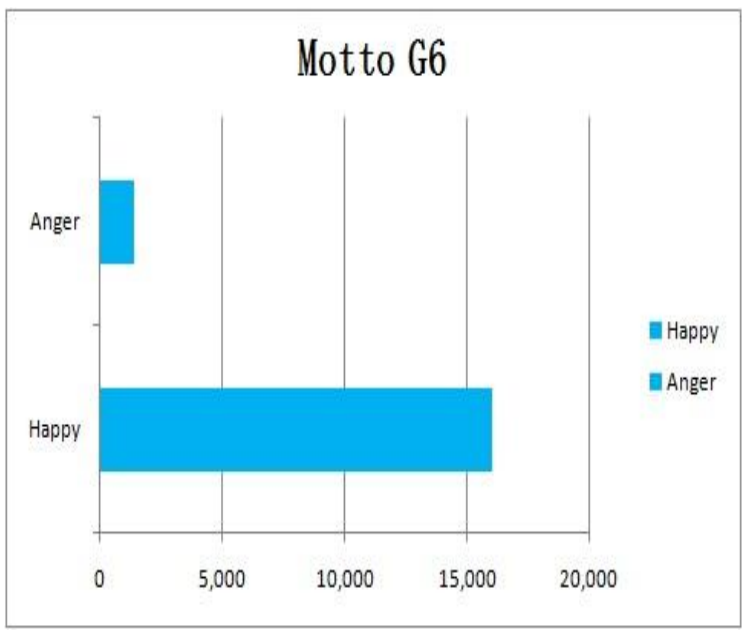

Fig 10: Positive and Negative reviews for Motto 


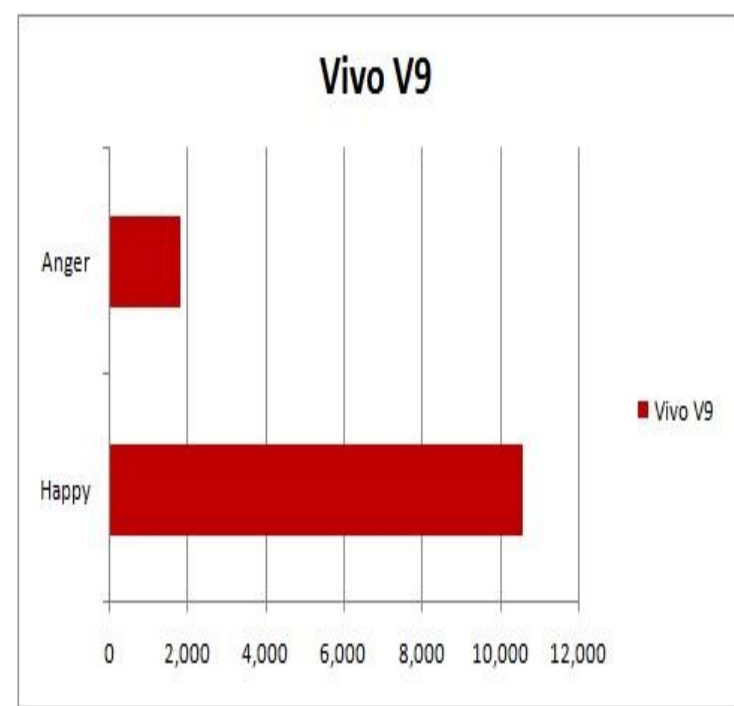

Fig 11: Positive and Negative reviews for Vivo

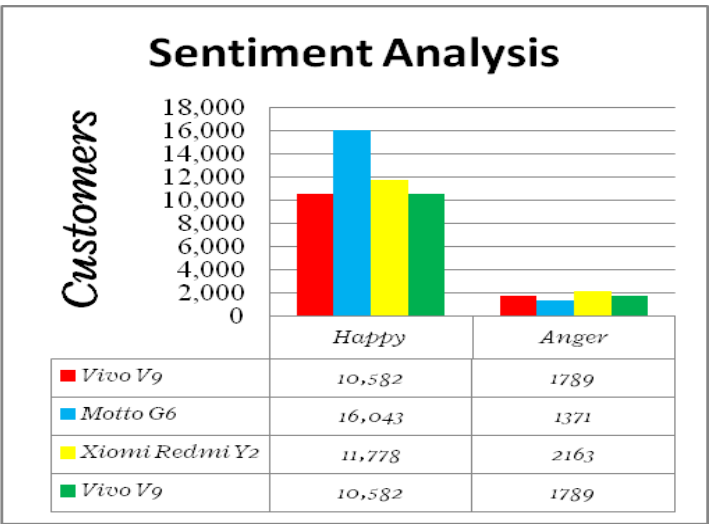

Fig 12: Combined Positive and Negative reviews of the brands.

The above figure 8, 9, 10, 11 explains only the positive and the negative reviews of the customers. The happiest and the sad reviews of the customers can be combined in a single comparative analysis.

\section{Cross Validation}

A sample of the named information is then prepared and tried on Support Vector Machine(SVM) classifier, whose execution is approve utilizing various cross approval. The execution of SVM is delineated in table II. The prescient precision of SVM turns out to be $86.68 \%$ which is very great.

TABLE I : CROSS VALIDATION FOR SVM

\begin{tabular}{|c|c|c|c|c|c|}
\hline Runs & $\mathbf{1}$ & $\mathbf{2}$ & $\mathbf{3}$ & $\mathbf{4}$ & $\mathbf{5}$ \\
\hline Accuracy & 87.34 & 89.98 & 87.45 & 86.22 & 82.43 \\
\hline Runs & $\mathbf{6}$ & $\mathbf{7}$ & $\mathbf{8}$ & $\mathbf{9}$ & $\mathbf{1 0}$ \\
\hline Accuracy & 80.12 & 89.98 & 89.43 & 88.22 & 85.65 \\
\hline
\end{tabular}

\section{CONCLUSION}

Fast change from the disconnected markets and custom to enlighten the purchasers regarding the strength of an item for development of internet business online markets has brought forth online surveys as the wellspring of trust building network. In this research the comparative analysis of the mobile product were discussed. The online review explores that the variation of the mobile products in various aspects of the brand. The SVM machine and the proposed clustering technique helps to segregate the reviews in a opinion view.

\section{REFERENCES}

1) M. Burke, C. Marlow, and T. Lento, "Social network activity and social well-being," in Proceedings of the SIGCHI conference on human factors in computing systems, 2010.

2) R. LiKamWa, Y. Liu, N. D. Lane, and L. Zhong, "Can your smartphone infer your mood?" ACM Workshop on Sensing Applications on Mobile Phones (PhoneSense), 2011.

3) M. De Choudhury, S. Counts, E. J. Horvitz, and A. Hoff, "Characterizing and predicting postpartum depression from shared facebook data," in Proceedings of the 17th ACM conference on Computer supported cooperative work \& social computing, 2014.

4) J. Reis, F. Benevenuto, P. Vaz de Melo, R. Prates, H. Kwak, and J. An, "Breaking the news: First impressions matter on online news," in Proceedings of the 9th International AAAI Conference on Web-Blogs and Social Media, 2015

5) International Journal of Software Engineering and Its Applications Vol. 9, No. 1 (2015), pp. 191-200 "Web Application for Sentiment Analysis Using Supervised Machine Learning"

6) Bryan Nii Lartey Laryea1, Chi-Hwan Choi, In-Sun Jung, Kyung-Hee Lee, "International journel of Advanced Research in Computer science and Software Engineering Vol. 5, No. 4 (2015), pp. 105-108 "Sentiment Analysis Using Support Vector Machine" Aamerza Z, Dr. Mohammad Atique, Dr. V M Thakare

7) D. Oelke, M. C. Hao, C. Rohrdantz, D. A. Keim, U. Dayal, L. E. Haug and Janetzko, "Visual opinion analysis of customer feedback data", IEEE VAST, (2009), pp. 187-194.

8) Li, Xiaodong et al., “ Market Impact analysis via sentiment transfer learning. Big data and smart computing( BigComp)",IEEE International Conference on IEEE,2017.

9) AI-Sharan,huda,et al,'Frame work for effective news analysis for Arabic news",International Conference on IEEE,2014.

10) Bouazizi et al.," Sentiment anlaysis: From binary to multiclass classification : A pattern based approach for multi class sentiment analysis from twitter".Communication (ICC), International Conference on IEEE,2016.

11) Kisan, Hase sudeep, Hasse Anand Kisan and Aher priyanka suresh, Collective Intelligence \& Sentiment anlaysis of twitter data by using Standford NLP Libraries with software as Service(SaaS)", Computational Intelligence and computing research (ICCIC), IEEE International conference, 2016

12) Lei et al.,Xiaojiang,,Xieuming qian and guoishua zhao," Cros language sentiment classification based on support vendor machine ". Natural computation (ICNC), IEEE International conference, 2015.

13) Desai,jairaj and swapnil r, andharia, " Sentiment analysis approach to adapt a shallow parsing based sentiment lexicon", Innovation in information, embedded and communication systems (ICIIECS), international conference, 2015.

14) Liu, Jie et al.," What makes a good review: Analyzing reviews on JD.Com", Service Science ( 
ICSS), IEEE International conference, 2015.

15) Selvan, Lokmanya thilak govindan sankar, teng sheng mong," A frame work for fast feedback opinion mining on twitter data streams collaboration technologies and systems(CTS), IEEE International conference, 2015.

16) Hagenau, Michael, micael liebmann anddirk neuman ," Automated news reading: Stock price prediction bassed on financial news using context capturing features", Decision Support System( 685697), 55.3,2013.

17) Xianghua, Fu et al.," Multi aspect Sentiment anlaysis for Chinese online social reviews based on topic modelling and hownet lexicon", Knowledge based systems (186-195), 37, 2013.

18) Moraes, Rodrigo, joao fransisco valiati and Wilson p gaviao neto, “ Document level sentiment classification : ana empherical comparision between SVM and ANN “, expert systems with applications (621-633), $40.2,2013$.

19) Soo-Min Kim and E. Hovy. Determining the sentiment of opinions. In Proceedings of COLING-04, 20th International Conference on Computational Linguistics, Geneva, CH, 2004, pp. 1367-1373.

20) Turney Peter. Thumbs Up or Thumbs Down? Semantic Orientation Applied to Unsupervised Classification of Reviews. In: Proceedings of the 40th Annual meeting of the Association for Computational Linguistics, 2002.

21) M. Hu and B. Liu. Mining Opinion Features in Customer Reviews [A]. In: Proceedings of Nineteenth National Conference on Artificial Intelligence (AAAI 2004) [C]. San Jose, USA, 2004.

22) Pang, Bo, L. Lee, and S. Vaithyanathan. Thumbs up? Sentiment Classification Using Machine Learning Techniques. In Proceedings of the Conference on Empirical Methods in Natural Language Processing (EMNLP), 2002, pp. 79-86

23) B. Pang, and L. Lee. A sentimental education: Sentiment analysis using subjectivity summarization based on minimum cuts. In Proceedings of ACL-04, 42nd Meeting of the Association for Computational Linguistics, Barcelona, ES: Association for Computational Linguistics, 2004, pp. 271-278.

24) Prem Melville, Wojciech Gryc and Richard D. Lawrence. Sentiment analysis of blogs by combining lexical knowledge with text classification. In Proceedings of KDD’2009. pp. 1275-1284.

25) Tao Li, Vikas Sindhwani, Chris H. Q. Ding and Yi Zhang. Knowledge transformation for cross-domain sentiment classification. In Proceedings of SIGIR'2009. pp.716-717

26) Rui Xia, Chengqing Zong, Shoushan Li. Ensemble of feature sets and classification algorithms for sentiment classification. Information Sciences, Vol.181(6), 2011, pp. 1138 - 1152.

27) Ainur Yessenalina, Yisong Yue and Claire Cardie. Multi-level Structured Models for Document-level Sentiment Classification. In Proceedings of the 2010 Conference on Empirical Methods in Natural Language Processing, MIT, Massachusetts, USA, 9-11 October 2010, pp. $1046 \mathrm{C} 1056$.

28) Schapire R. E., The strength of weak learnability, Machine Learning, 1990, 5(2), pp. 197-227. [13] Freund Y. Boosting a weak learning algorithm by majority. Information and Computation, 1995, 121(2), pp. 256-285.

29) Breiman L. Bagging Predictors. Machine learning, 1996, 24(2), pp. 123-40. [15] S. Li, C. Zong and X. Wang. Sentiment Classification through Combining Classifiers with Multiple Feature Sets. Processing and Knowledge Engineering, 2007.

30) Tsutsumi, K., Shimada, K. and Endo, T. (2007). Movie Review Classification Based on a Multiple Classifier. In Proceedings of the 21st Pacific Asia Conference on Language, Information and Computation (PACLIC21), pp. 481-488.

31) C.C. Chang, C.J. Lin. 2001. LIBSVM: a library for support vector machines. http://www.csie.ntu.edu.tw/ cjlin/libsvm/ [18] R. Xia, C.
Zong. 2010. Exploring the use of word relation features for sentiment classification. In Proceedings COLING- 2010.

32) R. Xia, C. Zong. 2011. A POS-based ensemble model for cross-domain sentiment classification. In Proceedings IJCNLP- 2011.

33) Tracking sentiment in mail: How genders differ on emotional axes, 2011.

34) R. D. E. F. X. Qi, K. Christensen, "A hierarchical algorithm for clustering extremist web pages," International Conference on Advances in Social Networks Analysis and Mining, 2010.

35) A. S. A. Naga, "A pagerank based detection technique for phishing web sites,” IEEE Symposium on Computers and Informatics, 2012.

36) [36] F. S. P. Sargolzaei, "Pagerank problem, survey and future research directions," International Mathematical Forum, 2010.

37) R. M. T. W. L. Page, S. Brin, "The pagerank citation ranking: Bring order to the web," Stanford Digital Libraries Working Paper, 1998.

38) J. Kleinberg, "Authoritative sources in a hyperlinked enviroment," ACMSIAM Symposium on Discrete Algorithms, 1998.

39) S.Muthukumaran, Dr.P.Suresh, "Text Analysis for Product Reviews for Sentiment Analysis using NLP Methods" International Journal of Engineering Trends and Technology (IJETT) - Volume 47 Number 8 May 2017, ISSN: 2231-5381, page No $474-480$ 